\title{
EDITORIAL
}

\section{Proteomics: improving biomarker translation to modern medicine?}

\author{
Paul C Guest ${ }^{*}$, Michael G Gottschalk and Sabine Bahn 1,2
}

Biomarkers are defined as 'measurable characteristics that reflect physiological, pharmacological, or disease processes' according to the European Medicines Agency [1]. The ideal platforms for biomarker discovery include genomic, transcriptomic, proteomic, metabonomic and imaging analyses. However, most biomarkers used in clinical studies are based on proteomic applications as the majority of current drug targets are proteins, such as G protein-coupled receptors, ion channels, enzymes and components of hormone signaling pathways [2]. Furthermore, linking the results of biomarker studies using protein-protein interaction approaches can assist in systems biology approaches and could lead to hypothesis generation and identification of new drug targets [3].

Proteomic-based approaches for biomarker investigation can be employed in different aspects of medicine, such as elucidation of pathways affected in disease, identification of individuals who are at a high risk of developing disease for prognosis and prediction of response, identification of individuals who are most likely to respond to specific therapeutic interventions, and prediction of which patients will develop specific side effects (Figure 1). In line with this, biomarkers can also be used for patient monitoring such as testing for 'normalization' of a biomarker signature in response to treatment or screening for re-appearance of a characteristic 'pathological' signature. All of this equates to improvement in patient care by using biomarkers in so-called personalized medicine approaches [4]. The progress and challenges in the translational application of proteomic technologies are highlighted in this new series, which features reviews written by leaders in the field on topics including post-translational modifications and proteinprotein interactions in disease.

Currently, there are only a few molecular tests that can predict response to certain treatments and these are

\footnotetext{
*Correspondence: pg110@cam.ac.uk

'Department of Chemical Engineering and Biotechnology, University of

Cambridge, Tennis Court Road, Cambridge CB21 QT, UK

Full list of author information is available at the end of the article
}

mainly restricted to the field of oncology. Perhaps the best example of this is human epidermal growth factor receptor 2 (HER2) expression in breast cancer cells. This cell surface receptor can be blocked by the antibodybased therapeutic Herceptin ${ }^{\mathrm{TM}}$ (trastuzumab) [5]. Such successes have raised hopes for discovery of biomarkers in other areas of medicine. However, in most cases, the claims for other novel biomarker candidates have not been proven in validation studies or in clinical trials. Potential reasons for this include deficiencies in design and analysis, the problem that drug targets and biomarkers may not be causal to the disease but rather a result of the disease process or a co-morbid effect, a lack of congruence of preclinical models with the human disease, or even because of factors such as the incorrect enrolment of patients in clinical trials who are too advanced in their disease stage to show any response to potential therapeutics [6]. Nonetheless, a consensus has now been reached for testing biomarker candidates in the earliest stages of a disorder, as described recently for neurodegenerative conditions such as Alzheimer's disease [7].

The suggestion that biomarker research has not lived up to the initial hype comes from the fact that publicized multiple 'breakthrough' tests have still not reached the market. This has led to skepticism from clinicians, scientists and regulatory agencies, which might make the introduction of valid biomarkers into clinical diagnostics or the drug discovery industry even more difficult. This is due in part to the lack of a connection between biomarker discovery with technologies for validation and translation to platforms that provide accuracy and ease of use in a clinical setting [8]. Apart from some biomarkers in the field of cancer research, most have not been validated and have now faded from the spotlight. Major cancer biomarkers that have received Food and Drug Administration (FDA) approval over the last few decades include prostate-specific antigen (PSA) for prostate cancer, carcinoembryonic antigen (CA)-125 for ovarian cancer and CA-19-9 for pancreatic cancer [9]. However, apart from the possible exception of PSA, most of these have been used mainly for monitoring treatment response and are not suitable for early diagnosis. 


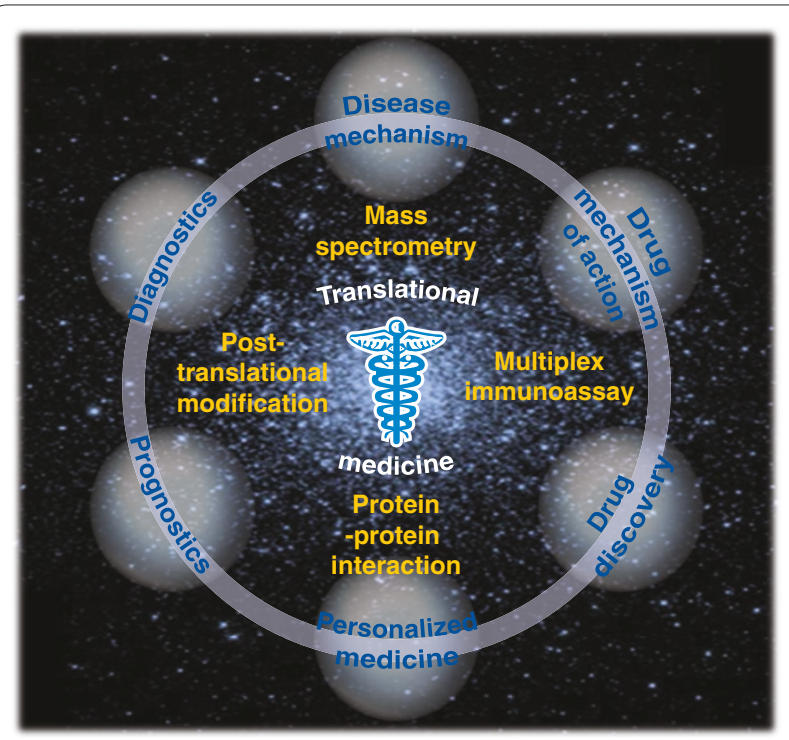

Figure 1. Ongoing and anticipated implementations of proteomic-based biomarkers in various aspects of medicine.

It has been suggested that the best strategy for biomarker qualification is through their co-development with drugs [10]. One of the best examples of this is the determination of the HER2 subtype of the epidermal growth factor receptor, combined with use of Herceptin $^{\mathrm{TM}}$, as described above. In this case, patients who have high levels of HER2 are more likely to respond to Herceptin ${ }^{\mathrm{TM}}$ treatment [5]. Thus, the use of scientifically and analytically validated biomarkers and rationally designed hypothesis-testing may lead to a paradigm shift in drug discovery and clinical trials. Researchers are now required to show that biomarkers are validated before they can be used in regulatory decision-making. According to the FDA, there are now three types of biomarkers based on proof of concept, validity and reproducibility (Table 1). The last category, which requires accurate replication of the findings, is where most promising biomarker candidates have fallen short. Currently, only long-standing and well-established tests have been used for regulatory decision-making, such as fasting glucose tolerance or glucose clamping to monitor insulin sensitivity [11].

It is clear that there is still a long way to go before the potential of proteomics can be entirely utilized in the preclinical and clinical fields, slowly progressing from bench to bedside and back again in an ongoing endeavor to improve patient outcomes. The tight regulations and concerted efforts outlined above will be essential in this journey. However, there is now optimism that further technological developments and interdisciplinary approaches will continue to advance the field of
Table 1. Three types of biomarkers for use in clinical studies

\begin{tabular}{ll}
\hline Biomarker type & Requirement \\
\hline Exploratory biomarkers & Evidence for scientific proof of concept \\
Probable valid biomarkers & $\begin{array}{l}\text { Measurement in an established analytical test } \\
\text { system and evidence explaining the clinical } \\
\text { significance of the results }\end{array}$ \\
Known valid biomarkers & $\begin{array}{l}\text { Biomarker test results should be accurately } \\
\text { replicated at different sites, laboratories or } \\
\text { agencies in cross-validation experiments }\end{array}$ \\
\hline
\end{tabular}

biomarkers so that its impact on modern medicine can be fully realized.

\section{Acknowledgements}

This work was supported by the Stanley Medical Research Institute (SMRI), the European Union FP7 SchizDX research program (grant reference 223427) and the NeuroBasic grant from the Dutch government. MGG is funded by a Gonville and Caius College - Cambridge Home and European Scholarship and an EPSCR doctoral training grant.

\section{Competing interests}

PCG and SB are consultants for Myriad-RBM.

\section{Author details}

'Department of Chemical Engineering and Biotechnology, University of Cambridge, Cambridge, UK. ²Department of Neuroscience, Erasmus Medical Centre, Rotterdam, The Netherlands.

Published: 27 February 2013

\section{References}

1. Biomarkers Definitions Working Group: Biomarkers and surrogate endpoints: preferred definitions and conceptual framework. Clin Pharmacol Ther 2001, 69:89-95.

2. Overington JP, Al-Lazikani B, Hopkins AL: How many drug targets are there? Nat Rev Drug Discov 2006, 5:993-996.

3. Sanz-Pamplona R, Berenguer A, Sole X, Cordero D, Crous-Bou M, Serra-Musach J, Guinó E, Ángel Pujana M, Moreno V: Tools for protein-protein interaction network analysis in cancer research. Clin Trans/ Oncol 2012, 14:3-14.

4. Honda K, Ono M, Shitashige M, Masuda M, Kamita M, Miura N, Yamada T: Proteomic approaches to the discovery of cancer biomarkers for early detection and personalized medicine. Jpn J Clin Oncol 2013, 43:103-109.

5. Pegram MD, Pauletti G, Slamon DJ: HER-2/neu as a predictive marker of response to breast cancer therapy. Breast Cancer Res Treat 1998, 52:65-77.

6. Flood DG, Marek GJ, Williams M: Developing predictive CSF biomarkers a challenge critical to success in Alzheimer's disease and neuropsychiatric translational medicine. Biochem Pharmacol 2011, 81:1422-1434.

7. Aisen PS, Andrieu S, Sampaio C, Carrillo M, Khachaturian ZS, Dubois B, Feldman $\mathrm{HH}$, Petersen RC, Siemers E, Doody RS, Hendrix SB, Grundman M, Schneider LS, Schindler RJ, Salmon E, Potter WZ, Thomas RG, Salmon D, Donohue M, Bednar MM, Touchon J, Vellas B: Report of the task force on designing clinical trials in early (predementia) AD. Neurology 2011, 76:280-286.

8. Plymoth A, Hainaut P: Proteomics beyond proteomics: toward clinical applications. Curr Opin Oncol 2011, 23:77-82.

9. Amayo AA, Kuria JG: Clinical application of tumour markers: a review. East Afr Med J 2009, 86(12 Suppl):S76-83.

10. Goodsaid F, Frueh F: Process map proposal for the validation of genomic biomarkers. Pharmacogenomics 2006, 7:773-782.

11. Khristov V: Glucose clamping - a modern method for research on insulin secretion and resistance. Vutr Boles 1986, 25:32-39.

doi:10.1186/gm421

Cite this article as: Guest PC, et al.: Proteomics: improving biomarker translation to modern medicine? Genome Medicine 2013, 5:17. 